Article

\title{
Elements of Infrastructure Demand in Multiplayer Video Games
}

\author{
Alexander Mirowski * and Brian P. Harper \\ Department of Informatics, Indiana University, Bloomington, IN 47408, USA; E-Mails: ajmirows@iu.edu (A.M.), \\ bpharper@iu.edu (B.P.H.) \\ * Corresponding author
}

Submitted: 30 June 2019 | Accepted: 18 October 2019 | Published: 20 December 2019

\begin{abstract}
With the advent of organized eSports, game streaming, and always-online video games, there exist new and more pronounced demands on players, developers, publishers, spectators, and other video game actors. By identifying and exploring elements of infrastructure in multiplayer games, this paper augments Bowman's (2018) conceptualization of demands in video games by introducing a new category of 'infrastructure demand' of games. This article describes how the infrastructure increasingly built around video games creates demands upon those interacting with these games, either as players, spectators, or facilitators of multiplayer video game play. We follow the method described by Susan Leigh Star (1999), who writes that infrastructure is as mundane as it is a critical part of society and as such is particularly deserving of academic study. When infrastructure works properly it fades from view, but in doing so loses none of its importance to human endeavor. This work therefore helps to make visible the invisible elements of infrastructure present in and around multiplayer video games and explicates the demands these elements create on people interacting with those games.
\end{abstract}

\section{Keywords}

eSports; infrastructure; infrastructure demand; multiplayer video games; video games

\section{Issue}

This article is part of the issue "Video Games as Demanding Technologies" edited by Nicholas David Bowman (Texas Tech University, USA).

(C) 2019 by the authors; licensee Cogitatio (Lisbon, Portugal). This article is licensed under a Creative Commons Attribution 4.0 International License (CC BY).

\section{Introduction}

Video games have long been sites of interaction and competition. A decade after the creation of one of the first video games-SpaceWar! (completed in 1962)devoted fans gathered to participate in an Intergalactic SpaceWar Olympics intended to prove whose skill with the prolific game was greatest (Brand, 1972, p. 1). Popular games such as Space Invaders and Tetris also saw tournaments spring up around them after their releases, mostly organized by enthusiasts in celebration of those games. Bowman's (2018, p. 16) four dimensions of demand in video games-cognitive, emotional, physical, and social-are readily visible in these early communal gaming spaces.

The advent of larger multiplayer environments in always-online games as well as organized eSports tour- naments represents an evolution of multiplayer video game play. The excitement of players and fans in interacting with favorite games still exists, as does their dedication to mastering those games. However, the nature of contemporary multiplayer video games produces an augmented set of requirements for players, developers, publishers, spectators, and other video game actors, due in large part to the infrastructure required to support what have become massive, multi-million-dollar global products, events, and experiences. Examining the elements of infrastructure in such games provides an opportunity to augment Bowman's (2018) conceptualization of demand in video games by identifying and exploring a new and complementary category of 'infrastructure demand' of games.

This article thus describes how the infrastructure increasingly built around video games creates demands 
upon those interacting with these games-as players, spectators, facilitators, and developers, among othersand we argue that these demands are particularly visible, and thus productively explicable, in contemporary multiplayer games. We follow the method described by Susan Leigh Star (1999, p. 380), who writes that infrastructure is as mundane as it is a critical part of society and as such is particularly deserving of academic study. When infrastructure works properly it fades from view, but in doing so loses none of its importance to human endeavor. This work therefore helps to make visible the invisible elements of infrastructure present in multiplayer video games, explicates the demands these elements create on people interacting with those games, and suggests the scholarly value of conducting such a deep dive into the infrastructural elements of multiplayer video games.

\section{Demand in Early Multiplayer Games}

The First Intergalactic SpaceWar Olympics, a small gathering of around two dozen enthusiasts, was held on October 19th, 1972, at the Stanford Artificial Intelligence Laboratory, and is emblematic of multiplayer video game play organized for the love of the game. The SpaceWar Olympics have been claimed as both the first video game tournament and the first eSports tournament ever held, but it is more defensible to say that it was the first well-documented video game tournament. The detailed account that we have of the event comes from a Rolling Stone article-"Spacewar: Fanatic Life and Symbolic Death Among the Computer Bums", written by the prolific Stewart Brand (1972), founder of the Whole Earth Catalog, its digital counterpart the Whole Earth 'Lectronic Link, and more recently co-founder of the Long Now Foundation. Though well-known for a variety of reasons, this chronicle of the SpaceWar Olympics serves for our purposes to highlight Bowman's (2018) four dimensions of demand-cognitive, emotional, physical, and social-as they are visible in small-scale communal video game play.

Bowman (2018, p. 11) describes physical demand as "the extent to which a system requires the user to exert discrete or holistic physical effort." This physical demand upon players of SpaceWar! and its Olympians is perhaps most apparent, with Brand (1972, p. 50) writing of their experience "locked in life-or-death space combat computer-projected onto cathode ray tube display screens, for hours at a time, ruining their eyes, numbing their fingers in frenzied mashing of control buttons"; there need be no debate that video game play is physiologically engaging, whether in 1972 or 2019. The cognitive demand upon players - "the extent to which the user is required to implicitly or explicitly rationalize or understand the game" (Bowman, 2018, p. 5)-is also evident, as Brand recounts the highly diverse strategies employed by each of the five players in one match, some taking advantage of mechanical firing techniques they developed and others leaning in to intimate knowledge of the gravitational well present in the arena. Some players in the Spacewar Olympics used mind games directed at their opponents to get the upper hand rather than superior mechanical skill, a fact that captures the social demand evident at the event, wherein the "system triggers an implicit or explicit response in the user to the presence of other social actors" (Bowman, 2018, p. 13). In the two-on-two event, Brand provides a rich account of team banter, ranging from "Good work" to evidence of sudden and perhaps inevitable betrayal-“NO! You killed me!" and the response, "Being partners means never having to say you're sorry." (Brand, 1972, p. 1). Emotional demand-"the extent to which a video game causes the user to have an implicit or explicit affective response to the game" -is perhaps less visible as directly originating from the game rather than from the tournament environment itself (Bowman, 2018, p. 8). However, the gameplay of Spacewar! was evocative, and we readily see this fourth category of demand in the tension, exultation, and frustration elicited by the Olympic competition itself as players interact with each other.

The Spacewar! Olympics were sponsored-by Rolling Stone-but only to the extent that Brand wanted to make sure the event was successful. In practice this meant providing beer and light snacks and funding the grand prizea year's free subscription to Rolling Stone. The reason Brand organized Rolling Stone's involvement, however, was not primarily to celebrate the game, but rather to highlight the wide-reaching technological change that he saw beginning to happen on university campuses and in government research labs across the United Statesthe laying of the groundwork of a new class of computer infrastructure (Baker, 2016). "Ready or not," he begins his article, "computers are coming to the people" (Brand, 1972, p. 1). Video games, Brand thought, were a particularly good case with which to see the benefits of this infrastructure: "They manifested so many amazing things about what was becoming possible with computers" (Baker, 2016), and competitive multiplayer video games even more so. How might this type of video game play, then, make visible a fifth demand of video gamesinfrastructure?

\section{Infrastructure Demand}

To illustrate the potential form and applications of the category of infrastructure demand, we turn to the work of Susan Leigh Star and others disentangling a different class of demanding technologies-the infrastructure that makes up our built world. There is much scholarship on the centrality of infrastructural concerns to organized video game play. Taylor and Witkowski (2010) explore the realm of LAN-enabled video game play-LAN standing for local area network-by examining LAN parties, gatherings of players to experience networked local multiplayer play; LAN parties are a prime example of the layered infrastructures, digital and not, that support video game play, and through participant observation they pro- 
vide rich accounts of how those infrastructures function together to support complex gaming spaces. Witkowski (2012) writes further about this complex interplay in an examination of Counter-Strike tournaments and interactions between the physical infrastructure supporting the game, such as keyboards and monitors, the infrastructure supporting players and their bodies, such as medical and fitness equipment, and the infrastructure of the game itself, such as the maps that matches are played upon and the visuals and sounds which communicate information to players at rapid speeds. Spilker, Ask, and Hansen (2018) examine of the layers of media infrastructure increasingly extant around video games; they describe Twitch.tv as a site in which audiences and media technologies intermingle, supported by the platform's infrastructure and in service to the enjoyment of games. Scully-Blaker, Begy, Consalvo, and Ganzon (2017) also write about Twitch, and through interviews they conduct with streamers on the platform we can see that the site itself is becoming infrastructure in its own right, and thus a critical part of an increasingly platformized gaming ecosystem.

Central to our formulation of infrastructure demand is Susan Leigh Star's (1999) methodological work in The Ethnography of Infrastructure. Star's approach in examining infrastructure is to invert the default perspective by focusing not on topics of systems, but on the "truly backstage elements" (Star, 1999, p. 380) that enable the network of systems to function. To this end, Star (1999, pp. 381-382) defines infrastructure by describing nine of its specific properties: embeddedness; reach or scope; built on an installed base; transparency; becomes visible upon breakdown; is fixed in modular increments, not all at once or globally; learned as a part of membership; links with conventions of practice; and embodiment of standards. Scholars of a variety of other systems have found application of these properties useful as a way to identify and open up the 'black box' of infrastructure and in so doing theorize that infrastructure's importance to systems at large. Dourish and Bell (2007) use the properties as a lens to examine the connection between sociality and ubiquitous computing as they relate to the organization and experience of space. Mark and Su (2010) apply them in examining changing work practices for 'nomadic workers,' individuals whose jobs require them to travel frequently. Arena, Arnaboldi, and Palermo (2017) make use of the properties to critically theorize enterprise risk management and boundary objects in accounting. Finally, Hartmann (2017) cites them as a useful starting point in asking questions about electrical power supply in an age of increasing smartphone use. We now suggest the utility of bringing Star's properties of infrastructure directly into discussions about video games, helpful as they are in conceptually breaking down otherwise complex and at times opaque systems. Discussions about the infrastructure of games, and the importance of that infrastructure, are not new. Bowman's conceptualization of demand in video games is new, however, and we be- lieve it can be productively augmented with the following explication of infrastructure demand: the extent to which a video game system requires other, organizing systems to function.

For our purposes, we shall summarize each of Star's properties and then contextualize them in the gaming and eSports space to better convey the breadth of this topic and the degree to which these infrastructural features place important demands upon gaming, sometimes boosting other sources of demand and sometimes creating their own unique demands.

\subsection{Embeddedness}

The systems most commonly thought of as infrastructure are those highly integrated into our society, like water, electricity, and transportation. These systems are connected to so many other systems that it is difficult to separate them cleanly from the other systems they rely upon. Electrical power has long been a requirement in video games, and this power consumption has a meaningful impact on electrical consumption globally, amplifying demands upon our climate and environment (Schatz, 2018). Internet access is now a mandatory infrastructural system to engage in most multiplayer video game experiences, and even in single-player experiences, as in the controversy over the always-online requirement of the 2013 game SimCity (Yin-Poole, 2013). Video games consoles have marketed themselves by making appeals to this embeddedness, as seen in the 6th generation of consoles (especially the PS2 and Xbox) portraying themselves as home entertainment systems that fulfill the role of DVD players. The current 8th generation from Sony and Microsoft (PS4 and Xbox One) are fully integrated into the video streaming landscape and let users access services like Netflix and Twitch. Video streaming itself, whether on Twitch, YouTube, Mixer, or other services, has become highly embedded into our esports landscape, with the ideas of a professional gamer and a professional streamer becoming blurred. Tournaments are streamed on these platforms, and many professional eSports competitors stream in addition to their eSports work, even to the extent of abandoning professional play to professionally stream (Nordmark, 2018).

\subsection{Reach or Scope}

Infrastructural systems stretch beyond single points in space and time and it does us little good to investigate specific moments of play without considering the larger systems at work. The moment of a major comeback in a tournament is not only an experience felt by those players, but also an experience mediated by all of the media systems watching that tournament. The commentators paid by the tournament contextualize the moment as it is watched physically by tournament attendees but also digitally by viewers on other platforms. The way in which these systems shape that moment is difficult to un- 
derstand by solely examining the experience of the competitors and requires a broader understanding of the demands present.

This property of infrastructure also prompts us to be wary of explanations of infrastructural systems that focus only on a specific case, as infrastructural demands, while far-reaching, will not be universal. Latency is a particularly visible expression of infrastructure in online games, but the efficacy of particular solutions will vary wildly based on the game in question and the user conditions. Sheldon, Girard, Borg, Claypool, and Agu (2003) argue that latency is not as strong a concern in real-time strategy games as in first-person shooter games, specifically using the case of Blizzard's Warcraft III. This finding was partially contradicted by Véron, Marin, and Monnet (2014), who investigated latency in multiplayer online battle arenas (MOBAs), a genre developed out of a mod for Warcraft III. The relevance of latency in both cases was dependent upon the use cases of these games more so than any innate property of the games. Sheldon et al. (2003) did not focus on high-level multiplayer play, and competitive play in the just-released Warcraft III had not developed to the point of requiring extremely high actions per minute, which would in turn require low latency. MOBAs have genre differences that contribute to a greater need for minimizing latency, but audience expectations are also important. The need to think about infrastructure is present in multiplayer games, but this need should also be well-contextualized to the state of the game and its users.

\subsection{Built on An Installed Base}

Infrastructure is not built in isolation; it is intended to be integrated with other systems. Console ownership is one of the few infrastructural concerns publicly addressed by the gaming industry, as games succeed or fail based partially on the success or failure of their platform(s). On an individual level, the experience of a game may be more or less enjoyable based on the infrastructural features of that platform (e.g., frames per second, field of view, control comfort, etc.). The social dimensions of games are also partially dependent upon what systems the game chooses to rely upon. The indie game Towerfall initially did not possess an online mode, featuring only local multiplayer. This choice, motivated likely by the cost to implement effective netcode and servers, shaped the social environment in which this game could exist, encouraging it to be viewed more like a party game than a competitive multiplayer game.

The PC marketplace is presently experiencing controversy based upon this installed base property. Valve's near-monopoly of the PC digital download space with their platform of Steam is being challenged by other companies, most notably with Epic's Epic Games Store. The pushback against this competition has been enormous, and while much of it can be attributed to noninfrastructural concerns, some of this criticism of Epic can be attributed to a lack of infrastructural features. As Grayson (2019) notes, "[the Epic Games Store is] as barren as they come in terms of features, with next to nothing in the way of community tools, cloud saves, achievements, wishlists, mod support, user reviews, forums, or other longtime Steam standards." These lacking features are not simply an issue for the storefront, as these features have become infrastructural concerns for the games themselves, especially for community interaction purposes. The lack of this expected infrastructure helps to explain the enormous pushback against Epic's competition.

Another issue highlighted by this property is the increasing difficulty of preserving video games. The infrastructural systems games are built upon are particular to certain points in time and space, which renders the creation of archives of video games highly challenging. Even ignoring the physical degradation of components, video games are created with a variety of infrastructural assumptions which may no longer be true. Necessary drivers might no longer exist, connecting cables might no longer be manufactured, necessary supplementary materials might be lost, etc. Even for more recent releases, compatibility issues represent considerable difficulty for consumers to play games as the infrastructure of software and hardware changes. CD Projekt's GoG.com (formerly Good Old Games) is able to find a place in the increasingly crowded game distribution marketplace partially because it provides support for legally buying and playing old games whose release versions will no longer run well on modern systems. Even this may not be sufficient for multiplayer games, however, as user configuration tweaks cannot revive needed dedicated servers or proprietary network protocols. Kaltman $(2016$, p. 2) writes about precisely this issue, suggesting that digital distribution and network contingent games are combining to create, for archivists, "an untenable nightmare" because of the need to increasingly preserve, restore, or emulate the infrastructure upon which games are built before effort can be spent on preserving the game itself; this is further complicated by the proprietary nature of much of this infrastructure, which adds legal questions to an already complicated endeavor.

\subsection{Transparency}

The usual explanation for the lack of attention paid to infrastructural concerns is that infrastructure is by design transparent. Though dozens of systems are needed for a viewer to tune into a Twitch stream of an eSports tournament, the viewer is interested not in those systems, but rather the match. Similarly, narratives of video game development often focus on designers who create new systems rather than the workers keeping those systems functional. This dynamic helps support the continued exploitation of workers within the video game industry, as is well demonstrated by periods of continual crunch that Epic Games has demanded of their workers to keep the 
game Fortnite functioning (Campbell, 2019). The lens of infrastructure is valuable for adding to discussions of the exploitative nature of the video games industry, both for examining abuses towards workers (Dyer-Witheford \& De Peuter, 2009) and for explorations of the exploitation of players as distributed labor (Ekbia \& Nardi, 2017).

Paradoxically, infrastructure and the work of specialists eminently aware of its inner workings often demands that it not be seen, and so despite the increase of maintenance work within the video game industry from the current trend towards live services, infrastructure remains usually invisible. Consequently, the work required to maintain and operate these systems is often unacknowledged, save when something goes wrong.

\subsection{Becomes Visible upon Breakdown}

When infrastructure systems fail, they suddenly become noticeable. Though it is not always clear which system has necessarily failed in the interconnected network of infrastructure, the existence of an infrastructure becomes immediately apparent. A tournament planned for months in advance may be disrupted by internet outages, as seen in a 2012 League of Legends tournament where the always-online nature of the game required multiple restarts of matches as the venue intermittently lost internet access (Tassi, 2012). Such interruptions inflict a psychological toll on competitors, especially as the typical transparency of infrastructural systems trains players to not worry about platforms such as Steam or Battle.net going down during a heated moment. Infrastructure failure can cause a spike in negative experiences as a result of increased demands of all dimensions upon players and the people supporting them. Despite the increased visibility of infrastructural systems upon failure, the interconnected nature of infrastructural systems does not allow for easy pinpointing of what part of the infrastructure has failed, and the work required to repair these systems remains specialized and difficult. Moments of breakdown are useful to critically examine, however, as they provide unique windows into the inner workings of otherwise hidden systems and structures.

\subsection{Is Fixed in Modular Increments, Not All at Once Or Globally}

As befits the interconnected nature of infrastructural systems, it is not possible to correct breakdowns in infrastructure in totality. Fixes are always made to pieces of these systems, which send ripples throughout the rest of the network. The simplistic request common on gaming forums for the developers to 'fix the netcode' is not possible without changing many other variables. Some code will always be older than other code, and games will always rely upon pieces of infrastructure best described either figuratively or literally as crumbling. As in many cases of infrastructure work, the effort of maintainers is not well-rewarded, especially in the exploitative envi- ronment of the video game industry (Dyer-Witheford \& De Peuter, 2009). The proper maintenance of video game infrastructure, therefore, is another type of demand that affects players and developers alike.

The gradual development of video game infrastructure renders investigations of past iterations of online games challenging. The game itself will undergo regular patches that change features, and this regular iteration can make the state of the same game years apart radically different. While games like World of Warcraft have dedicated communities that record the patch history of the game, even this fails to account for unannounced or undocumented changes to the game. In some cases, old versions of the game simply do not exist any longer. The history of Final Fantasy XIV is an example of this challenge. This game was radically redesigned following an unsuccessful first release, and the development along the 1.X branch prior to the $2 . X$ rerelease is difficult to study, let alone to play, even for those with access to the developers themselves (O’Dwyer \& Jayne, 2017).

\subsection{Learned as A Part of Membership}

An important consideration for the development of social demands in games is not just the experience of play, but the experience of learning infrastructural norms from other players. For children, learning the rules and norms of a new Minecraft server is not just a social requirement for fitting into a new group of people, but an infrastructural requirement for them to understand how and when they are permitted to use server commands and what lines of authority have been set up to deal with infrastructural concerns. It is also possible to understand the development of so-called 'metas' agreed upon norms of acceptable and/or optimal play choices-in competitive games as an infrastructural demand. While social demands surround the choice to use or not use meta playstyles, a meta is a type of infrastructure that becomes learned by joining and becoming part of a community of players, and there are often social consequences for ignoring or pushing against these norms. A longer discussion of the infrastructure of the meta, part technological and part social, is presented below.

\subsection{Links with Conventions of Practice}

Moving further away from physical notions of infrastructure, the norms and conventions of the game industry can also be considered infrastructure. Design elements are limited by the conventional practice of the game industry, providing the benefits of 'intuitive' (or more accurately, 'conventional') interface and control schema, but also limiting design choices. Sometimes these limitations impose additional physical demand on players by forcing them to use challenging control systems to play a game that violates some of these conventions, as when a controller layout mandates the use of certain straining hand postures to optimally play. This problem may sim- 
ply be a physical demand of the game, but it is also interpretable as a unique infrastructure demand for playing a game that is integrated into a certain infrastructural system. Perhaps switching to a different control system would alleviate these concerns, but support for such differences is another infrastructural requirement that the developer would have to incorporate.

The Nintendo Switch provides an interesting object of investigation for this subject, as its position as a hybrid console/handheld platform links it to two (or perhaps three) different lineages of conventions. While there are many different design implications from this, the choice of control system is perhaps the most explicit. The options to play in the handheld or docked modes, coupled with the choices of attached Joy-Cons, detached Joy-Cons, or a Pro controller reveal differing ideas about how games are supposed to be played. Designers have interesting limitations when designing for the Switch, as these different conventions necessitate either the rejection of certain conventions or the accommodation of multiple options at once. Games like Tetris 99 can be linked to a particular convention (original Tetris controls) and argue for a certain control scheme (detached Joy-Cons) being closer to the conventional ideal, but this is a negotiated and social process.

\subsection{Embodiment of Standards}

Likewise, the choices made to create a game in a certain way are often the embodiment of other infrastructural standards that are forced upon developers. The choice of platform, game engine, or user interface is often dictated by the standards of larger bodies like console manufacturers or publishers. While a game engine provides affordances for developers, it also embodies the standards of its creator, and if an engine is licensed from someone else, there may be conflicts between these standards and those desired by the developer. This problem is harshly stated by Schreier (2019) discussing the development of Anthem: "Many of the features those developers had taken for granted in previous engines, like a saveload system and a third-person camera, simply did not exist in [the Frostbite engine]." While the narrative of the failure of Anthem is complex, the game engine embodying different standards of game development was an infrastructural problem that put considerable demands on development time and resources. Those infrastructure demands for developers can then become demands for players, as the creation of fixes to resolve a calcified development problem often falls to players. Thus, a game can create demands for players before it is even played.

\section{Overwatch League}

What sort of work can be done with an eye towards infrastructure demand? Blizzard Entertainment's Overwatch League (OWL), an eSports institution, provides a good example case to demonstrate the value of narratives told within the framework of infrastructure demand, specifically of a case where infrastructure demands push developers in contradictory directions. Overwatch is a first-person shooter whose standard mode of play sees teams of six players face off against each other to complete various objectives on maps set in a fictionalized Earth by playing some combination of three hero roles-Damage, Tank, and Support. Blizzard announced the formation of the OWL at Blizzcon 2016, a convention dedicated to the company's games. Summerley $(2019$, p. 5) notes that this was in fact the same year that that the game itself launched, making the game and League a unique and useful "case where a developer has managed almost every aspect of a game's journey into a sport including rapid commercialization and professionalization" and, of interest to us, the development of its infrastructure. The scholarship examined earlier reveals the infrastructural issues that affect organized competitive video game play on stage and at events dedicated to the practice. Certainly, the OWL, as an example of a large, well-funded competitive program, evinces such issues as well. But what more can be revealed if we follow the threads made visible to us with the lens of infrastructure demand? In what other ways does infrastructure matter in the competitive video game ecosystem, and why should scholars of games be interested in asking these questions? The following case will illustrate.

Our point of entry is the concept of the meta, introduced earlier. Metas within games are themselves infrastructure, working to construct norms and assumptions about what 'proper' gameplay is; various metas have developed in Overwatch over the course of the game's existence, and it is in the story of one of the most recentGOATS-that we can examine thornier questions of infrastructure demand. This is a case about the breakdown of infrastructure, which not only renders it visible but also uncovers the web of embedded systems and practices that Star tells us to expect when taking a deep dive into such studies. The case of OWL demonstrates a situation in which the infrastructure demands of Overwatch as eSport do not match the demands of Overwatch as game played by a large and diverse player base. This dissonance between the two demands has made itself known in struggles around the mechanical balance of the game as well as questions about who the game is for; an important point to be made about infrastructure demand is that it is variable based upon who experiences it.

The game of Overwatch offers two major play modes-'Quick Play' and 'Competitive.' The latter is a ranked mode of play that assigns a visible numerical skill rating (SR) to players based upon their performance in the mode, taking into account wins and aptitude with their chosen heroes. The SR ranges between 1 and 5000 , and within that range exist different ranks (named 'Bronze,' 'Silver,' 'Platinum,' 'Diamond,' 'Master,' and 'Grandmaster'), usually at every increment of 500 . The efficacy of various strategies and heroes in the game varies significantly across ranks, as does the population 
of players. In February of 2018, Overwatch Lead Designer Jeff Kaplan revealed the percentage of Overwatch players who had attained each rank with an image showing that $62 \%$ of the game's player base had an SR lower than the numerical halfway point of 2500 (Kaplan, 2018). By comparison, OWL players, if they play the Competitive mode, are almost certain to attain a rank that puts them in the top $1 \%$ of players, according to Kaplan's (2018) post.

Herein lies our infrastructure question: For whom should the game be designed? It might seem reasonable for Overwatch's developers to spend much of their time balancing the game's mechanics and supporting systems around the majority of the player base to ensure an enjoyable experience. But OWL requires that the game, when played by some of its most skilled players, also be enjoyable to watch, since spectatorship is critical in sustaining any professional sport. There exists a tension here, because the game is a single artifact and the same rules and mechanics of the game apply whether a player is on stage or at home, in Silver or Grandmaster. This situation is a perennial problem in eSports games, and reconciling this tension is still a significant challenge, and one with which Blizzard has made some unusual choices. OWL attempts to solve this problem by selectively choosing when new versions of the game are used by the tournament, thus allowing professional players to play one version of the game while non-professional players play another. This choice undermines the sense of Overwatch as a specific game and has led to player complaints about enjoyment watching OWL, since it often meant an old version of the game was being played (Czar, 2018). Central here is the point that the same single design decision regarding a particular hero, map, or fundamental mechanic in the game will create different and perhaps irreconcilable demands for different groups in the OWL and Overwatch community.

This tension is perhaps most visible in the variety of changes to the game made by Blizzard in an attempt to move away from one particular dominant strategic composition of heroes-GOATS, which consists of three Tank heroes and three Support heroes. It is outside the scope of this article to tell the whole story of GOATS, but it is sufficient to say that its use in high level OWL play was extremely effective, best defeated with mirrored use of the GOATS composition, difficult to successfully emulate by the majority of the Overwatch player base due to its complexity, and generally agreed by OWL viewers to be decidedly uninteresting to watch (Van Allen, 2019). GOATS mirror matches became the norm in OWL, to the detriment of professional players of Damage heroes, who had no place in GOATS, and viewers, who knew exactly what to expect from each match. While other factors were at play, the primacy of GOATS certainly contributed to the declining viewership numbers that OWL was experiencing at the time, despite the incredible resources Blizzard was investing in spreading OWL to as many platforms as possible, from its own client to Twitch, China's Zhanqi.tv, and on ESPN (Miceli, 2019).
Blizzard alternately attempted to displace the dominant GOATS by: (1) weakening some of the heroes in the composition to open up opportunities for more than the six heroes in GOATS to be seen on stage at one time; (2) changing the mechanics of one type of health in the game upon which GOATS heavily relied-armor-in an attempt to open up counterplay; and most recently (3) restricting the number of each role of hero that can be played on a team in Quick Play and Competitive while also preventing the switching of heroes across different roles during play. This last change, known as 2-2-2 because it limits all teams to two heroes of each role, has been the most dramatic in reconfiguring the way the game functions and in determining how its most popular modes can or cannot be played. Overwatch players had also been asking for this change outside of the context of OWL, since the structure imposed by 2-2-2 was believed to solve other issues with the game, but it is inarguable that part of its implementation was a direct challenge to GOATS, which had become ingrained in OWL and feeder competitions for the League.

Conceptualizing these changes to the game as infrastructural fixes, we can become aware of the ripple effects they have had upon the game and in turn its players. The act of adjusting the power of heroes (the first changebalancing) carries implicit assumptions on the part of developers about how those heroes are used by players; that use is not uniform across the player community or across ranks. This means that demands on players vary as they learn how to play and play against newly balanced heroes; changes that alter the meta in one way in one rank can alter it in other ways at other ranks, and this was the case as balance fixes cascaded through the game. The second act-changing a core game mechanic-brought with it more extreme results. Armor reduces the amount of damage a hero would take with a very specific mathematical formula. Change the formula, and suddenly interactions involving any hero with armor change as well. Determinations of which hero is strong versus another shift, which necessitates changes in strategy, team compositions, and the social dynamics associated with choosing those compositions at the start of a match. All are a form of infrastructure, and all feel the effects of a change in just one. The long-term results of the third act-changing the structure of the teams-are still to be seen at time of writing. We can read in Blizzard's official announcement of the change a sensitivity to multiple types of demand at play in Overwatch:

A lot of important decision-making happens in the 40 seconds before a match even begins, as everyone selects their heroes and responds to others' role choices. It's not uncommon for players - who may all have different goals and play styles - to feel tension, pressure, disappointment, or even hostility as a team composition comes together. The Role Queue system is designed to help take the edge off this process, ultimately leading to matches that feel fairer and more 
fun, where players are in roles that they want to be in. (Blizzard Entertainment, 2019)

While marketed as a purely positive change, limiting the number of each hero role in each team has shaken standard conventions of practice within the game. While shaking the infrastructural meta for OWL was the point of this change, this alteration was applied broadly, with aftershocks felt by the rest of the game's players. Anecdotally, many players, at home and in the League, believe that 2-2-2 removes a core element of the game-the ability to dynamically and creatively determine the composition of one's team to outmaneuver one's opponents-and in so doing will fundamentally weaken it. Some players who identified themselves as 'flex' players, those skilled in switching roles fluidly before and during a match as they determined situations dictated, saw the infrastructure of the game change to eliminate their preferred practice. Their mode of play is no longer possible in the game's Quick Play and Competitive modes and many of these players have felt alienated as a result. It has yet to be seen what long-term effect 2-2-2 will have on OWL's success; the enjoyment of OWL spectators, and the livelihoods of OWL commentators and professional players depend on it, fundamental as it is to Overwatch's gameplay. Such is the nature of infrastructure; there may be 'backstage elements' yet to reveal themselves as this case unfolds.

\section{Conclusion}

This conceptualization of infrastructure demand may also help elucidate broader concerns for video games. While Blizzard may conceptualize their investments in expanding the viewership of Overwatch onto new platforms as a sort of infrastructure investment, thinking of changes to their game systems as infrastructural shifts would help in recognizing when their decisions demonstrate infrastructural conflict. The infrastructure they are building for OWL, whether in game versions or in sweeping balance changes, conflicts with the infrastructure already established for non-professional play. The problems Blizzard is trying to solve are not isolated issues; they are part of interconnected infrastructure, and changes to these systems will have effects on other systems, both social and technological.

In the previous sections, we communicated through multiple levels of analysis that there are many ways to apply the concept of infrastructure demand to video game research. Its value comes from its broad applicability to experiences at multiple levels and in multiple modes of interaction with video games. These include player experiences, which are the primary focus of the four types of demand laid out above-cognitive, emotional, physical, and social-but also the experiences of game developers, esports facilitators, and more, which are shaped by infrastructure demands that may not be visible at first glance. Within the context of video games as a source of demand, infrastructure may inform our understanding of existing sources of demand by proposing new interpretations, as in the example of physical demand and controllers, while also enabling the exploration of new questions of demand, such as considering the environmental cost of video games, which would be difficult to conceptualize under a purely individualistic understanding of the study of video games. This conceptualization may also benefit designers of games by allowing for a broad and interconnected understanding of the systems requisite for a game to function. Thinking of the meta of a video game as infrastructure is not only a semantic adjustment. It makes tangible what otherwise might be thought of as arbitrary social conventions. Not only do these demands upon a game have weight, but attempts to change them must also be taken with care, as they are still connected to other systems; one well-intentioned solution to a problem might cause a break in numerous other places.

\section{Acknowledgments}

We would like to thank the anonymous reviewers for their comments and suggested revisions for the article, which helped to hone the arguments herein. We would also like to thank the editors for their assistance in the submission and revision process.

\section{Conflict of Interests}

The authors declare no conflict of interests.

\section{References}

Arena, M., Arnaboldi, M., \& Palermo, T. (2017). The dynamics of (dis)integrated risk management: A comparative field study. Accounting, Organizations and Society, 62, 65-81.

Baker, C. (2016). Stewart Brand recalls first 'Spacewar' video game tournament. Rolling Stone. Retrieved from https://www.rollingstone.com/culture/culturenews/stewart-brand-recalls-first-spacewar-videogame-tournament-187669

Blizzard Entertainment. (2019). Introducing role queue. Blizzard. Retrieved from https://playoverwatch.com/ en-us/news/23060961/introducing-role-queue

Bowman, N. D. (Ed.). (2018). Video games: A medium that demands our attention. Oxford: Routledge.

Brand, S. (1972, December 7). SPACEWAR: Fanatic life and symbolic death among the computer bums. Rolling Stone.

Campbell, C. (2019). How Fortnite's success led to months of intense crunch at Epic Games. Polygon. Retrieved from https://www.polygon.com/2019/4/23/ 18507750/fortnite-work-crunch-epic-games

Czar, M. (2018). The patch problem in Overwatch League. Daily Esports. Retrieved from https://www. dailyesports.gg/the-patch-problem-in-overwatch- 
league

Dourish, P., \& Bell, G. (2007). The infrastructure of experience and the experience of infrastructure: Meaning and structure in everyday encounters with space. Environment and Planning B: Planning and Design, 34(3), 414-430.

Dyer-Witheford, N., \& De Peuter, G. (2009). Games of empire: Global capitalism and video games. Minneapolis, MN: University of Minnesota Press.

Ekbia, H. R., \& Nardi, B. A. (2017). Heteromation, and other stories of computing and capitalism. Cambridge, MA: MIT Press.

Grayson, N. (2019). Why people are so mad about the Epic Games Store. Kotaku. Retrieved from https:// kotaku.com/why-people-are-so-mad-about-theepic-games-store-1833848770

Hartmann, M. (2017). Circuit(s) of affective infrastructuring. In J. Vincent \& L. Haddon (Eds.), Smartphone cultures (pp. 11-24). Oxford: Routledge.

Kaltman, E. (2016). Current game preservation is not enough. How They Got Game. Retrieved from https://web.stanford.edu/group/htgg/cgi-bin/ drupal/?q=node/1211

Kaplan, J. (2018). Competitive mode tier distribution. Blizzard. Retrieved from https://us.forums. blizzard.com/en/overwatch/t/competitive-modetier-distribution/972

Mark, G., \& Su, N. M. (2010). Making infrastructure visible for nomadic work. Pervasive and Mobile Computing, 6(3), 312-323.

Miceli, M. (2019). Overwatch League Twitch viewership continues to slide as stage three starts. The Esports Observer. Retrieved from https://esportsobserver. com/owl-twitch-stage-three-2019

Nordmark, S. (2018). Live streamer or competitive gamer-Which career makes the most sense? Dot esports. Retrieved from https://dotesports.com/ general/news/esports-vs-streaming-money-career31144

O'Dwyer, D., \& Jayne, J. [Noclip]. (2017, June 23). Final Fantasy XIV documentary part \#1-“One point $O$ " [Video file]. Retrieved from https://www.youtube. com/watch?v=XsOyQKI7Yw4

Schatz, B. (2018). Video games consume more electricity than 25 power plants can produce. Mother Jones. Retrieved from https://www.motherjones.com/ environment/2018/11/video-games-electricitycarbon-footprint

Schreier, J. (2019). How BioWare's anthem went wrong. Kotaku. Retrieved from https://kotaku.com/howbiowares-anthem-went-wrong-1833731964

Scully-Blaker, R., Begy, J., Consalvo, M., \& Ganzon, S. C. (2017). Playing along and playing for on Twitch:
Livestreaming from tandem play to performance. In Proceedings of the 50th Hawaii International Conference on System Sciences (pp. 2026-2035). Honolulu, $\mathrm{HI}$ : University of Hawaii.

Sheldon, N., Girard, E., Borg, S., Claypool, M., \& Agu, E. (2003). The effect of latency on user performance in Warcraft III. In Proceedings of the 2nd Workshop on Network and System Support for Games (pp. 3-14). New York, NY: ACM.

Spilker, H. S., Ask, K., \& Hansen, M. (2018). The new practices and infrastructures of participation: How the popularity of Twitch.tv challenges old and new ideas about television viewing. Information, Communication \& Society. Advance online publication. https:// doi.org/10.1080/1369118X.2018.1529193

Star, S. L. (1999). The ethnography of infrastructure. American Behavioral Scientist, 43(3), 377-391.

Summerley, R. (2019). The development of sports: A comparative analysis of the early institutionalization of traditional sports and e-sports. Games and Culture. Advance online publication. https://doi.org/10. $1177 / 1555412019838094$

Tassi, P. (2012). Internet meltdown at LoL world championships shows eSports has a problem. Forbes. Retrieved from https://www.forbes.com/sites/ insertcoin/2012/10/08/internet-meltdown-atlol-world-championships-shows-esports-has-aproblem

Taylor, T. L., \& Witkowski, E. (2010). This is how we play it: What a mega-LAN can teach us about games. In Proceedings of the Fifth International Conference on the Foundations of Digital Games (pp. 195-202). New York, NY: ACM.

Van Allen, E. (2019). What is GOATS in Overwatch, and why are fans so upset with it? Polygon. Retrieved from https://www.polygon.com/2019/2/25/ 18239845/overwatch-goats-meta-triple-tankexplainer

Véron, M., Marin, O., \& Monnet, S. (2014). Matchmaking in multi-player on-line games: Studying user traces to improve the user experience. In Proceedings of Network and Operating System Support on Digital Audio and Video Workshop (p. 7). New York, NY: ACM.

Witkowski, E. (2012). On the digital playing field: How we "do sport" with networked computer games. Games and Culture, 7(5), 349-374.

Yin-Poole, W. (2013). Maxis attempts to explain why SimCity is always-online as players discover code for 20-min offline force-shutdown timer. Eurogamer. Retrieved from https://www.eurogamer. net/articles/2013-03-18-maxis-attempts-to-explainwhy-simcity-is-always-online-as-players-discovercode-for-20-min-force-shutdown-timer-for-offline 


\section{About the Authors}

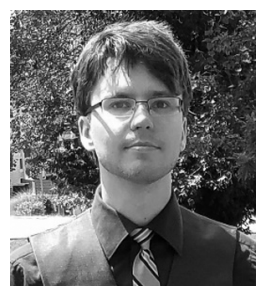

Alexander Mirowski is a PhD Candidate in the Informatics Department of the Luddy School of Informatics, Computing, and Engineering at Indiana University. He is a historian of technology, and in particular of video games. He is also the founding director of the Informatics Game Research Group at IU, whose mission is to bring together scholars across disciplines to study games and to contribute to the continued growth of game studies.

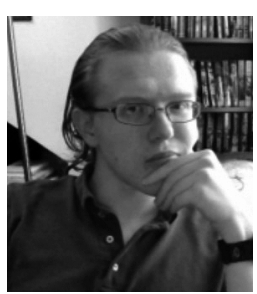

Brian P. Harper is a PhD Student in the Informatics Department of the Luddy School of Informatics, Computing, and Engineering at Indiana University. He studies social media from STS, political economy, and genre studies perspectives, with a particular emphasis on social media adjacent to gaming, such as Let's Plays on YouTube and streaming on Twitch. 